\title{
El puerto de Barcelona en la primera mitad del siglo XVIII. Urgencias estructurales e infraestructurales a toda costa durante el reinado de Felipe $\mathbf{V}$
}

\section{Juan Miguel Muñoz Corbalán}

Universitat de Barcelona, Barcelona, España, juanmiguelmunoz.corbalan@ub.edu

\begin{abstract}
The Barcelona Harbor played an important role as a part of the Spanish defensive system from the beginning of the XVIIIth Century. Among all interventions by military engineers can be highlighted the fight against sedimentation of sand and mud, the design of retaining structures, the configuration of the harbor, the problem of the lantern, the artillery defense, the arrangement of the beach, the struggle between quarters and buildings linked to maritime activity, and the need of building a new customs office.
\end{abstract}

Keywords: military engineering, hydraulic engineering, military architecture, 18th-century harbors

\section{Introducción}

La consolidación de la estructura del Estado tras la Guerra de Sucesión condujo a la administración borbónica a plantear una revisión en el diseño de las fronteras marítimas. Barcelona, enclave trascendental en el esquema estratégico del reino, necesitaba una serie de reformas que favorecieran el control del territorio pero también un sistema de comunicaciones eficaz para hacer viable el desarrollo nacional. Los proyectos y las intervenciones en el puerto de la Ciudad Condal de mano del Cuerpo de ingenieros militares fueron trascendentales para modernizar la propia dinámica urbana, afianzar su seguridad defensiva y consolidar la organización del litoral español en base a una distribución equilibrada de sus sectores navales.

Circunstancias como las prioridades iniciales en torno al acondicionamiento del puerto, su definición estructural, los equipamientos indispensables, la conservación del fondeadero, la integración en el sistema defensivo de la ciudad, la necesidad de sincronizar el progresivo desarrollo industrial y comercial de Barcelona con la obligada transformación del conjunto portuario y las correspondientes infraestructuras aduaneras, etc., contribuyeron a cimentar las bases de una realidad urbana acorde con el despegue socioeconómico promovido institucionalmente desde la Corona y otros organismos públicos y privados, a la vez que servían como retos profesionales y formativos para los ingenieros que debían responsabilizarse de su proyección y ejecución ${ }^{1}$.

\section{Primeras acciones}

Tras la victoria borbónica frente al opositor austríaco en la Guerra de Sucesión, los estamentos de gobierno de la monarquía observaron la necesidad de convertir la Ciudad Condal en un seguro enclave estratégico para el control del territorio. Mientras que Ferrol, Cádiz/Málaga y Cartagena (junto a los presidios norteafricanos) constituían tres puntos capitales para la organización de las defensas costeras en términos de frontera, la ausencia de un puerto en 
el cuadrante nororiental del reino que reuniera las condiciones adecuadas para poder equipararse, principalmente, a los ejemplos gallego y murciano, condujo a centrar el foco de las reformas en el puerto barcelonés, el cual requería unas intervenciones de alcance para cumplir dichos objetivos.

Siguiendo las directrices especificadas en la Instrucción, y Ordenanza de 4. de Julio de 1718 del Cuerpo de ingenieros, redactadas por el propio Ingeniero General, pronto se hizo necesario acometer la empresa de mejora del puerto de la Ciudad Condal y la organización del nuevo Barrio de la Playa. Entre otras, las razones que movieron la agilización de la iniciativa fueron, según el Capitán General del Principado, que los habitantes del sector demolido del Barrio de La Ribera que se habían quedado sin vivienda con motivo de la erección en sus terrenos de la ciudadela barcelonesa se iban asentando en Mataró y en otras poblaciones de la costa cercanas a Barcelona, «en grave perxuicio del comercio de este puerto y de las operaciones del Real servicio que en él pueden ofrecerse» ${ }^{2}$. Sin embargo, los proyectos para el Barrio de la Playa y el de las Huertas de San Pablo, de finalidad similar, firmados por el flamenco en abril de 1721, tampoco formaron parte de las prioridades de la Secretaría de la Guerra hasta que, años más tarde, el 31 de diciembre de 1751, fue retomada dicha posibilidad de la mano de Juan Martín Zermeño, dentro de un Proyecto general para fortificar a Barcelona, Ciudadela, Monjuyc y mejorar el puerto ${ }^{3}$.

\section{La transformación del puerto}

De forma simultánea a las propuestas para la erección de la ciudadela de Barcelona, la segunda empresa a la que hubo de enfrentarse Jorge Próspero durante su estancia en la capital del Principado tras la expedición de Sicilia en 1719-1720 fue el puerto de Barcelona, ante la necesidad de efectuar una serie de mejoras para su buen funcionamiento ${ }^{4}$. Como parte de una iniciativa general para perfeccionar la seguridad y la operatividad de los puertos de mar en las costas del reino fueron necesarias las inspecciones y descripciones de todo el perímetro marítimo peninsular. Antes de partir para Andalucía con la misión de llevar a cabo las reformas pertinentes en los puertos de Málaga y Cádiz, frente a la degradación sufrida por la fuerza de los vientos y las aguas, Verboom dio comienzo a las labores de mejora del puerto de Barcelona con una serie de sondas realizadas durante el mes de febrero de 1721 para determinar la profundidad de sus aguas y la naturaleza de los sedimentos acumulados en su fondo. En 1742, Joseph Francisco de Alós, máximo responsable del consistorio de Barcelona, remitía al Secretario de la Guerra una sucinta memoria sobre sucesivos proyectos confeccionados para solucionar los graves problemas del puerto barcelonés ${ }^{5}$. En ella, el miembro del cabildo municipal reiteraba la urgencia de solucionar los retrasos que se habían venido produciendo desde el primer proyecto de 1721, concebido por el Ingeniero General, con sendas revisiones realizadas por él mismo en 1731 y 1741. Alós mencionaba, además de uno «antiguo que proponía zerrar el Puerto desde la linterna al Montjuí, dexando la entrada a la parte del Leveche», planteado en 1583 e inviable por sus características y su coste ${ }^{6}$, la sucesión de tres proyectos en 1721, «sin que se hayan podido ver ni examinar otros planos ni ideas para la defensa total del Puerto que las expresadas».

La sinopsis del regidor barcelonés era muy elocuente para mostrar la supuesta simplicidad de la toma de decisiones a seguir con el fin de decidir entre los dos del italiano Spirito Pascali y «Uno del año 21. hecho $\mathrm{p}^{\mathrm{r}}$ el Marq ${ }^{\mathrm{s}}$ de Berbon y anadido [sic] despues en el de 31. y 41. consistiendo en dos prolongacion ${ }^{\mathrm{s}}$ una à la Isquierda de la Linterna del Puerto de cien tuesas declinada à la parte del Sudueste para detener y embarazar las arenas q arrastran las corrientes furiosas del este, y otra en la parte de la derecha inclinada al sur, dexando entre estas dos prolonga $^{s}$ ó Brazos capacidad para una ensenada ó receptaculo de las arenas, quando venciesen la punta del Brazo izquierdo, las q pudieran sacarse con Pontones par $^{\mathrm{q}}$ no superasen la punta del Brazo ó prolong ${ }^{\mathrm{n}}$ de la derecha, la q cubrirà el Puerto en parte de los impetus del viento sur y le mantendrà con fondo suficiente, una vez levantadas las arenas que actualm ${ }^{\text {te }}$ le tienen 
perdido». La idea del flamenco consistía en una propuesta de «Augmentacion del Muelle para mejorar este Puerto è impedir que no [sic] se ciegue» ${ }^{7}$.

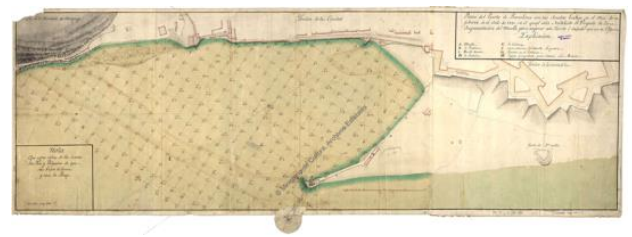

Fig. 1- Jorge Próspero Verboom et al. (s.f.): Plano del Puerto de Barcelona con sus Sondas hechas en el Mes de febrero de el Año 1721 en el qual está Señalado el Proyecto de la Augmentacion del Muelle para mejorar este puerto è impedir que no [sic] se Ciegue. S.1. [Barcelona], s.a. [ca. $10 \mathrm{de}$ mayo de 1721]. AGS.MPD, 02,011.

El proyecto de Verboom presentaba una prolongación desde la batería al final del muelle menor y su linterna en forma de doble espigón: por un lado el dique orientado hacia mediodía, con la función de alargar el muelle, siendo coronado con una nueva batería; por el otro, el dirigido hacia poniente, más corto, con la intención de «detener las Arenas». En realidad, este plan ya había sido elaborado por Jorge Próspero en 1719 como respuesta a una idea de Spirito Pascali que el italiano había esbozado con anterioridad a la expedición de Sicilia. La propuesta del flamenco para configurar un puerto "abierto", como también manifestó en su plan malagueño, venía tras observar el rápido y progresivo deterioro del fondeadero, de manera que «desde que he vuelto a esta plaza que su puerto se va perdiendo por instantes, pues desde que partí para Sicilia se ha ido considerablemente ensanchando un pedazo de playa que se ha formado delante la muralla de la plaza a la derecha de la Puerta del Mar, que es el cabo del referido puerto, donde se recojen las barcas de los pescadores, y a donde ha venido tanta arena, sobre una buena distancia puerto adentro, que casi ya no queda fondo en aquel parage, lo que ocasionan los vientos de mediodía y leveche, que son los de la travesía de este puerto que llevan a la playa las arenas de un banco que se ha formado en todo el largo de la boca de él, desde la linterna al pie de Montjuich, con las que los levantes y las corrientes trahen para lo largo de la costa, de las que salen de la boca del río Bezós al mar, quando hay crecidas (...); he hecho sondar bien todo el puerto y su entrada, que e hallado de muy diferente y mucho menor fondo que tenía tres años ha» ${ }^{8}$.

Jorge Próspero insistía en el interés de hacer un proyecto urgente por la importancia que dicha obra tenía para «el Real servicio y al bien público (...) así por la situación del puerto tan a propósito para todo, sin haver otro en toda la costa desde los Pirineos asta Alicante, como por el público beneficio del comercio de aquel Principado y de las rentas que produce a V.M.». El tiempo de ejecución estimado por el flamenco era de «tres veranos, que corresponden a dos años y medio de trabajo» ${ }^{9}$. Las primeras labores, correspondientes a «tomar las sondas y medidas de él, y sus contornos», tuvieron complicaciones relacionadas con «el mal tiempo, que ha sido continuo asta la fin de la semana passada», aunque era urgente que pudieran ser finalmente acabadas «a fin de incluirlo todo en el plan y mapa de él, a lo que se está trabaxando para añadir después el proyecto» ${ }^{10}$.

El Ingeniero General dejó listas la Descripción del Puerto de Barcelona y la Estimación del coste el 8 de mayo de 1721. Dos días más tarde remitía toda la documentación manuscrita y el plano correspondiente (fig. 1). Las reflexiones de Jorge Próspero intentaban no dejar cabos sueltos y justificar con lógica las decisiones técnicas tomadas ${ }^{11}$. Ante su inminente partida hacia Andalucía, Verboom dejaba en manos del Ingeniero Director Alejandro de Rez «los borrones e instrucciones de todo para poner este proyecto en execución, quando el Rey lo aprobare». El análisis realizado por el flamenco partía de la base de que, con anterioridad, el puerto era accesible para navíos y fragatas de 50 a 60 cañones. Tras las sondas, la evidencia era que solamente un pequeño sector ante la cabeza del muelle viejo disponía de características aproximadas, con tres brazas de profundidad, mientras que tales embarcaciones requerían de cuatro a cinco brazas. Tampoco era operativo el caladero próximo al baluarte de San Ramón y a la Puerta de Mar, único paraje protegido del viento de levante. Su escasa profundidad 
impedía el atraque de los barcos. De hacerlo en lugares más hondos (en la travesía del puerto hacia el mediodía) el riesgo radicaba en que el Levante los empujara hacia la muralla urbana o las rocas al pie de Montjuïc. Para afrontar dichos inconvenientes Jorge Próspero hubo de buscar el origen del problema. La lógica a partir de la observación metódica de los diversos factores le llevaron a inferir que los bancos de arena que obstaculizaban el paso y el atraque de las embarcaciones se formaban con el Levante y las corrientes marinas procedentes de las costas de Mataró, junto con las arenas y el cascajo de las avenidas del río Besós, así como las aguas del Rec Comtal, la acequia procedente de los molinos del Clot, con sus tierras e inmundicias desembocando al mar entre el Fuerte Don Carlos y la linterna del muelle.

Sin embargo, Verboom detectaba otras cuatro causas para la degradación del puerto barcelonés. La primera, el descuido en su limpieza y mantenimiento, «especialmente desde el último bloqueo y sitio de esta plaza, que estubo más de dos años sin limpiarse, y después acá se ha trabajado poco en ello». En segundo lugar, los fuertes temporales del invierno de 1715-1716, «que se llevaron un pedazo de muelle nuevo cercano del viexo, por cuia brecha, que se dexó algún tiempo sin componer, entró porción de piedras y maior de arenas en el puerto». La tercera causa tenía que ver con «otras inmundicias y Lodos por cinco desagües urbanos al mar, sobre todo con las lluvias». En último lugar, la escasez de material para el dragado, puesto que «sólo hay dos pontones, insuficientes para el ritmo de sedimentación $»^{12}$.

El flamenco evaluaba los criterios seguidos hasta el momento de cara a atajar los problemas y juzgaba críticamente las diversas opiniones y discursos manejados por los entendidos en la materia para acabar con las corrientes de arena y los detritos del Rec Comtal, una de cuyas actuaciones habría de consistir en la construcción de un contramuelle frente a la ciudadela, a la altura del Fuerte Don Carlos, un poco más costa arriba o en la boca de la acequia. La obra que acabó realizándose fue la prolongación del muelle hacia el mar, «lo que se acabó de ejecutar algunos 32 años ha que se concluyó la porción que ay desde el muelle viexo $\mathrm{B}$ asta el nuevo $\mathrm{D}$, de que se experimentó durante algún tiempo un gran beneficio en dicho puerto hasta los nuevos sedimentos». La reflexión de Verboom para considerar equivocada la medida por su poca continuidad en el tiempo se basaba en que «al dilatar el mencionado muelle, lo herraron [sic] en inclinarle tanto a la parte de poniente, que si le huvieran prolongado en línea recta desde la cabeza del muelle viexo acia el sureste o siroco, huviera sido más durable, porque se huviera detenido más tiempo la arena fuera de la boca del puerto». Las tres medidas propuestas por Jorge Próspero para remediar los males y contribuir al sostenimiento de lo llevado a cabo eran: «formar un espigón de bloques grandes de piedra de Montjuïc, que servirá para detener las arenas que en este caso huvieren doblado la referida cabeza (...) también para mejor cubrir contra los temporales de las embarcaciones que se encontraran en el puerto»; emplear la arena acumulada a modo de playa «a levante del muelle viexo» como material de construcción «en las fabricas de casas y demás edificios» de la ciudad; y «eliminar el banco seco que suele formarse al poniente de la linterna».

El flamenco tasó las obras a realizar en 45.000 doblones. Tal y como venía siendo habitual, el enorme volumen de trabajos simultáneos por todo reino dificultó el correcto desarrollo de lo previsto. Spirito Pascali, encargado de dirigir las faenas de limpieza del puerto durante el largo período de tiempo que Jorge Próspero se halló lejos de Barcelona, había sido el autor de las otras dos propuestas (una de puerto cerrado hasta Montjuïc, con bocana en su medianía, y otra de muelle prolongado y rematado por un martillo) y mostraba su disgusto por el desprecio del que había sido objeto, comentando al ministro que «los ingenieros de acá puede ser que digan a V.E. que este espigón (que propongo como un prompto remedio para que este puerto no acabe de morir mientras se alargue el muelle, y se haga el martillo propuesto) no conviene. Yo me alegraría, y toda la gente del mar quedarían admirados, si proponían a V.E. otro espediente más acertado, más breve y de tan poco coste» ${ }^{13}$. 
Este resentimiento irónico hacia la posición de Verboom y sus subordinados era manifestado por el Conde de Glimes en una defensa de Pascali y de «los molinetes de que ha hecho experiencia en este puerto y los que despreciaban esta invención ${ }^{14}$. Dicha situación mostraba una vez más el turbio ambiente existente entre las autoridades gubernativas locales (Capitanía General e Intendencia del Ejército) y los miembros del Cuerpo de ingenieros.

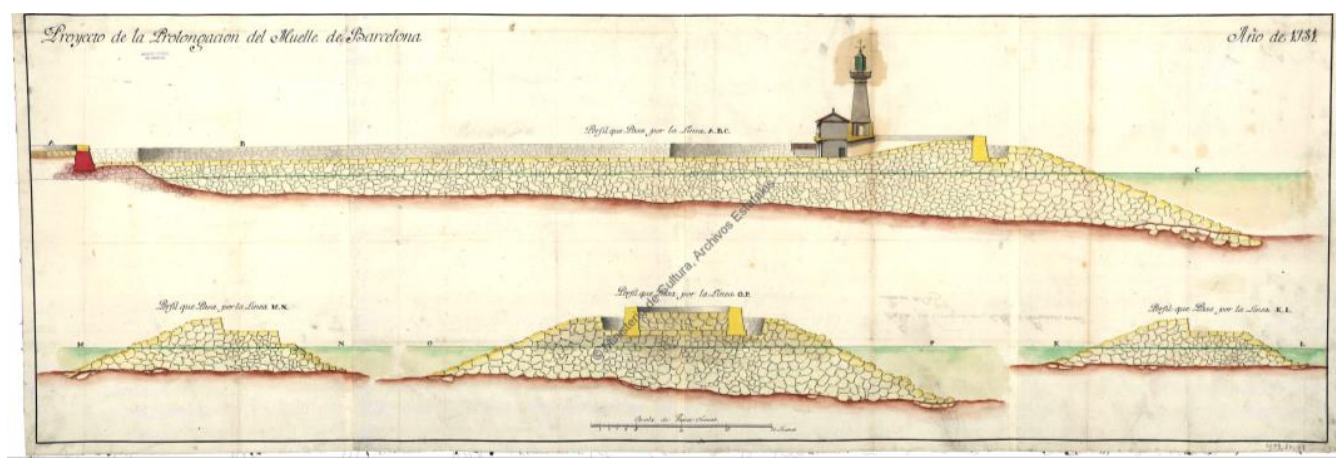

Fig. 2- Perfiles del Proyecto de la Prolongacion del Muelle de Barcelona. Año de 1731. S.f. [¿Miguel Marín?]; s.l. [Barcelona], s.a. [1731]. AGS.MPD, 20,037.

El italiano, insistiendo en las excelencias de su proyecto de 1730 (aprobado pero detenido en 1733 por la «guerra de Italia», conflicto bélico insertado en la Guerra de Sucesión de Polonia y, tras la victoria de las tropas españolas en Nápoles en verano de 1734, en su modificación mediante un nuevo plan «sobre el estado al que se haya al presente, que he puesto en manos de este Intendente, el qual toda la gente de mar y pilotos de este puerto confían ser el único modo de bien restablecerlo», intentaba asumir la responsabilidad de la empresa ${ }^{18}$. Por su parte, el flamenco, quien ya había podido comprobar el fracaso de propuestas supuestamente eficaces como las del holandés Jacob Van Daalen en Cádiz, constataba la falta de fondos para proseguir regularmente las obras. Según los criterios de Jorge Próspero, el principal remedio era la prolongación del muelle, pero ello no sería eficaz sin la continuación del trabajo de dragado, para lo cual había solicitado aumentar el número de pontones con el fin de transportar los sedimentos del fondo, labor que seguía siendo dirigida por Pascali, «quien ha sacado un espigón de 9 toesas de largo en la medianía del muelle dentro del puerto, suponiendo que con esto lo impedirá, sin considerar que los temporales le inutilizaran luego su obra, que con las arenas quedará enterrada (...) con lo qual se frustra también su idea de que dicho espigón sirva de embarcadero» ${ }^{16}$.

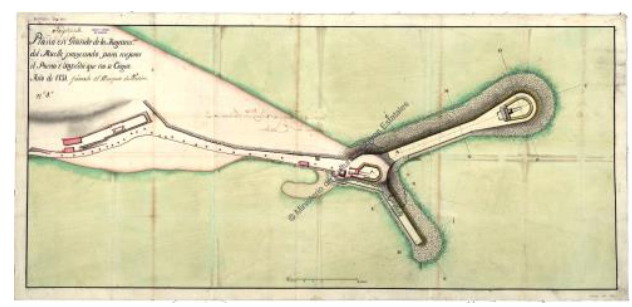

Fig. 3- Plano en Grande de la Augmenton del Muelle proyectado para mejorar el Puerto é impedir que no [sic] se Ciegue. Año de 1731. Firmado el Marques de Verbôm. S.f. [Juan Baltasar Verboom et al.]; s.l. [Barcelona], s.a. [ca. primavera de 1742]. AGS.MPD, 20,036.

Los años fueron sucediéndose sin ser resuelto el estancamiento del proceso, aunque la intención subyacente era poder seguir la planificación firmada por Jorge Próspero en 1721. Pedro Superviela, miembro de la Real Junta de Fortificaciones creada en Madrid en 1737 y fiel delineador de aquél durante muchos años, al ser requerido para elaborar un informe sobre los diferentes proyectos puestos sobre la mesa, los dos de Pascali y los de Verboom de 1721 y 1731 (fig. 2-3) ${ }^{17}$, concluyó que el puerto "abierto" del 
italiano, iniciado en su momento, resultó «completamente inútil por haverse enterrado la obra con la primera borrasca que hubo, introduciéndose las arenas al pie del andén. como antes, como lo previno el Marqués de Verbom» ${ }^{18}$. Ya en un delicado estado de salud a mediados de los años 30, el Ingeniero General no podía afrontar personalmente la problemática relativa al puerto de Barcelona.

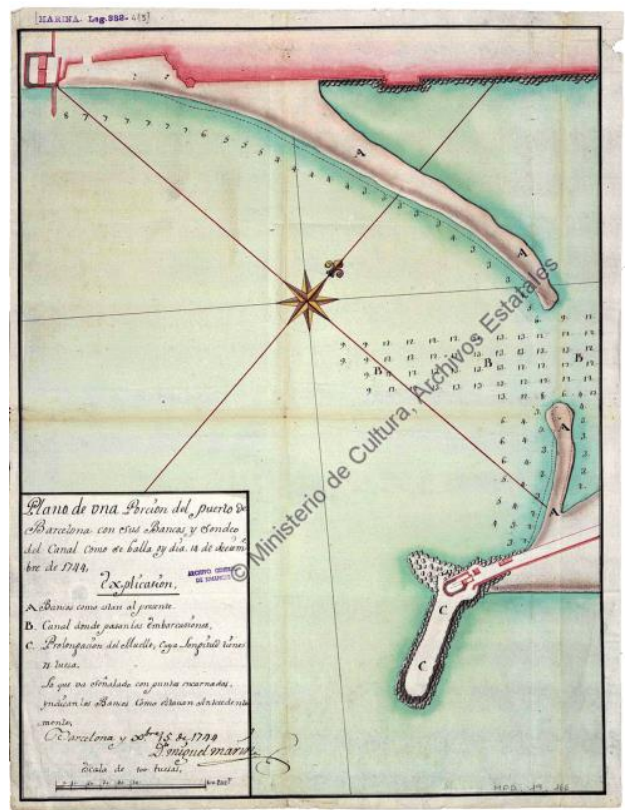

Fig. 4- Miguel Marín (f.): Plano de una Porcion del puerto de Barcelona con sus Bancos y Sondeo del Canal como se halla oy día. 14. de diciembre de 1744. Barcelona, 15 de diciembre de 1744. AGS.MPD, 19, 166.

Ya en manos de Miguel Marín la Dirección de ingenieros de Cataluña, el marsellés (quien disponía de amplia experiencia en obras portuarias en Francia) ${ }^{19}$, se responsabilizó de las labores a realizar en él, ejecutando numerosas sondas periódicas del fondo y plasmando sobre el papel el estado de los bancos de arena formados en el puerto con sus progresivos cambios (fig. 4) ${ }^{20}$. Sin embargo, desencuentros entre varios ingenieros (el propio Marín frente a Jaime Sicre, Juan Martín Zermeño y, sobre todo, Fernando La Sale, Francisco Antonio Framboisier, Joseph Dufresne, José Vallejo y el miembro de la Junta de Fortificaciones Juan de
La Ferrière) provocaron serios incidentes personales e institucionales que contribuyeron notablemente al desconcierto en el desarrollo de los trabajos en el puerto entre 1740 y 1751, año en que Marín abandonó la dirección del Principado.

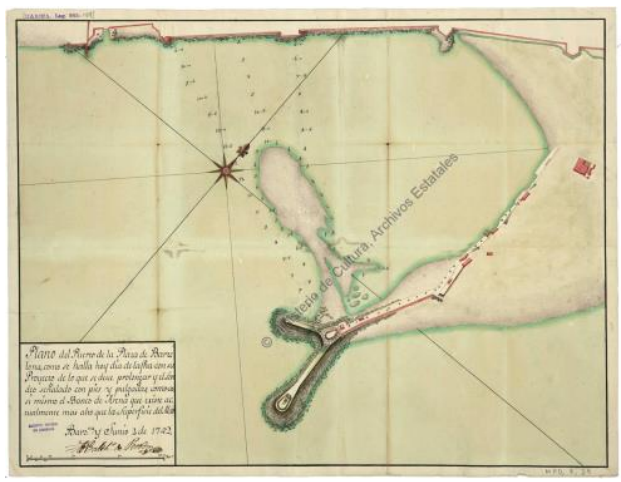

Fig. 5- Juan Baltasar Verboom (f.) et al.: Plano del Puerto de la Plaza de Barzelona, como se halla hoy día de la fha. Con su Proyecto de lo que se debe prolongar y el Sondeo señalado con pies y pulgadas, como assí mismo el Banco de Arena que existe actualmente mas alto que la Superficie del Mar. Barcelona, 2 de junio de 1742. AGS.MPD, 07,039.

Hasta el fallecimiento de Jorge Próspero en enero de 1744, y durante la ausencia del francés en 1742, fue su hijo Juan Baltasar Verboom quien actuó como mediador de la figura de su padre en un proceso donde éste ya no podía intervenir, tal y como quedó patente en la supervisión de los sondeos del puerto (fig. 5) ${ }^{21}$ y a raíz de sendos requerimientos desde la Corte para estudiar y dictaminar en lo referente al plan de Miguel Marín «sobre la limpia, concervasión y fortificación de este puerto», encargos que el Ingeniero General no fue capaz de cumplir sin delegar absolutamente en Juan Baltasar, quien por estas fechas, como su padre, también se hallaba en un delicado estado de salud ${ }^{22}$.

Marín aprovechó la oportunidad que le brindaba la definitiva indisposición de Jorge Próspero Verboom y la de su hijo Juan Baltasar para proponer una planificación más amplia del sistema formado por la prevista prolongación del propio muelle (aunque modificada en tamaño y estructura), la transformación del edificio de la 
Lonja en un cuartel de mayor capacidad y nuevos edificios para la ciudadela. En el sector portuario, el marsellés proyectaba, también retomando ideas lanzadas por el flamenco varios años atrás, aunque sin llegar a la envergadura de los existentes en Cartagena y Ferrol, un completo arsenal de fragatas y galeras, con los correspondientes astilleros y diques de carenado Este diseño de arsenal de marina anulaba la posibilidad de utilizar el terreno de la playa entre la ciudadela y el muelle en uso para crear en él el antiguo proyecto concebido por el Ingeniero General de un Barrio de la Playa donde alojar a una buena parte de los expropiados con motivo de la edificación del fuerte abaluartado tras la limpieza de los terrenos afectados en el Barrio de La Ribera. La idea de Miguel Marín contemplaba la erección de una imponente linterna para ser ubicada en el extremo del espigón orientado al Mediodía, además de una potente batería artillera de planta elíptica (fig.6).

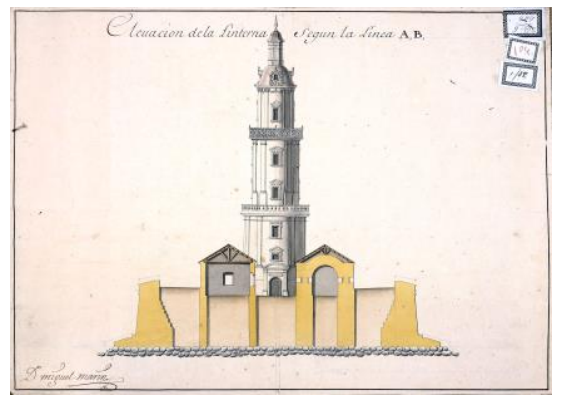

Fig. 6- Miguel Marín (f.): Elevación de la Linterna Según la línea A,B. S.1. [Barcelona], s.a. [ca. 1740]. AGM,M.CH, B-10/10.

El fallecimiento de Jorge Próspero Verboom en 1744, con los subsiguientes cambios en la comandancia del Cuerpo de ingenieros y la dirección del Principado, así como la transmisión del trono español a Fernando VI tras la muerte de su padre Felipe $\mathrm{V}$ dos años más tarde, abrieron una nueva etapa en la política de intervenciones planificadas para el puerto de Barcelona, el cual, unas décadas más tarde, pudo resolver algunos de los problemas que habían venido impidiendo durante mucho tiempo su adecuación a las demandas de la propia Corona $\mathrm{y}$ al progresivo desarrollo social, comercial y económico de la Ciudad Condal.

\section{Notas}

(1) Esta aportación forma parte de un estudio de mayor envergadura en el que me hallo trabajando bajo el título El puerto de Barcelona en el siglo XVIII. De Pallas bellica a Minerva hydraulica o la labor del ingeniero militar "como pez en el agua". Ambas quedan integradas en el proyecto del Ministerio de Economía y Competitividad HAR2012-31117 "El Dibujante Ingeniero al servicio de la Monarquía Hispánica. Siglos XVI-XVIII", dirigido por la profesora de la UNED Alicia Cámara Muñoz.

(2) Carta del Marqués de Castelrodrigo a Miguel Fernández Durán; Barcelona, 8 de septiembre de 1718. AGS.SGU, $3323-8^{\circ}-3^{\mathrm{a}}$-a.

(3) Montserrat Galera, Francesc Roca y Salvador Tarragó: Atlas de Barcelona. Segles XVI-XX. Barcelona: Col-legi Oficial d'Arquitectes de Catalunya, 1982 ( $2^{\mathrm{a}}$ edic.), pp. 214-221. Juan Miguel Muñoz Corbalán: "Cartografía militar y representación espacial de Barcelona en el siglo XVIII", en Carme Montaner y Francesc Nadal (ed.): Aproximacions a la història de la cartografia de Barcelona. Barcelona: Institut Cartogràfic de Catalunya / AHCB, 2011, pp. 3145. Juan Manuel Alfaro Gil: Juan Martín Zermeño. La ingeniería militar al servicio de la Ilustración (1713-1773). Tesis doctoral (dir. Juan Miguel Muñoz Corbalán). Barcelona: Universitat de Barcelona, 2013.

(4) Joan Alemany i Llovera: El Puerto de Barcelona. Un pasado, un futuro. Barcelona / Madrid: Lunwerg, 2002 ( $2^{\text {a }}$ edic.).

(5) Breve Apuntacion de los Proyectos que desde el año de 1719. hasta ahora se han hecho por medio de sus planos y perfiles para precaver la ruina del Puerto de Bar ${ }^{n a}$ y liberarle de la copiosa entrada de Arenas y fango, y sobre los quales informò el director $G^{l}$ Marq $q^{s}$ de Berbon à S.M. en los años de 1721, 1731 y 1741. Joseph Francisco de Alós; Barcelona, 20 de octubre de 1742. AGS.SM, 381.

(6) Joan Alemany i Llovera: Els fars de Catalunya. Tiana: Rosa Esteve i Associats, 2006, pp. 46-48. 
(7) Carta de Jorge Próspero Verboom al Marqués de Castelar; Barcelona, 8 de mayo de 1721. AGS.SM, 382/16.

(8) Carta de Jorge Próspero Verboom al Marqués de Castelar; Barcelona, 8 de marzo de 1721. AGS.SM, 382/5.

(9) Íd.

(10) Carta de Jorge Próspero Verboom al Marqués de Castelar; Barcelona, 21 de abril de 1721. AGS.SM, 382/2.

(11) Descripcion del Puerto de Barcelona en el estado en que se halla (...). Jorge Próspero Verboom; Barcelona, 8 de mayo de 1721; y Estimacion del coste que tendrá el prolongar cien tuesas mar adentro el muelle de piedra del Puerto de Barcelona (...). Jorge Próspero Verboom; Barcelona, 8 de mayo de 1721. AGS.SM, 382/16.

(12) Carta de Jorge Próspero Verboom al Marqués de Castelar; Barcelona, 8 de mayo de 1721. Íd.

(13) Carta de Spirito Pascali al Duque de Montemar; Barcelona, 21 de mayo de 1735. AGS.SM, 382/31.

(14) Carta del Conde de Glimes a José Patiño; Barcelona, mayo 1735. AGS.SM, 382/30.

(15) Carta de Spirito Pascali al Duque de Montemar; Barcelona, 2 de febrero de 1737. AGS.SM, 382/31.

(16) Carta de Jorge Próspero Verboom a José Patiño; Barcelona, 21 de mayo de 1735. AGS.SM, 382/28.

(17) Vid., también, Proyecto de la Prolongacion del Muelle de Barcelona. S.f.; s.l. [Barcelona], s.a. [1731]. AGS.MPD, 08,160.
(18) Memoria de Pedro Superviela, s.d.; s.l., s.a. AGS.SM, 382/32.

(19) Juan Miguel Muñoz Corbalán: "La linterna de Barcelona. El proyecto «clasicista» de Miguel Marín en 1740", en Actas del X Congreso del CEHA Los Clasicismos en el Arte Español (Comunicaciones). Madrid: Universidad Nacional de Educación a Distancia Departamento de Historia del Arte, 1994, pp. 537-547.

(20) Vid., entre muchos otros, Miguel Marín et al. (s.f.): Plano de porcion del Muelle, y Puerto de Barzelona, en que se demuestra, el banco de Arena que se ha formado nuevamente. Barcelona, 28 de febrero de 1733. AGS.MPD, 19,147; y Miguel Marín (f.) et al.: Plano del Puerto de la Plaza de Barcelona, y su sondeo, en el Estado que se halla al presente, con su proyecto formado, para darle mayor Capacidad, e impedir se introduzcan en el, las arenas que trahe el Río Besòs. Barcelona, 18 de marzo de 1740. AGS.MPD, 13,047.

(21) Vid., también, Miguel Marín (f.) et al.: Plano del Puerto de la Plaza de Barcelona, donde esta señalado el banco de arena que se ha formado ultimamente con su sondeo hecho en 12. de Nobiembre de este presente año y en dho. Plano esta puesto el proyecto dela Prolongacion del Muelle con su paredon para impedir la entrada de las arenas en dho. Puerto. Barcelona, 17 de noviembre de 1741. AGS.MPD, 02,018.

(22) Carta de Jorge Próspero Verboom a José del Campillo; Barcelona, 9 de diciembre de 1741. AGS.SM, 382/94. Minuta de despacho s.f. [José del Campillo] a Jorge Próspero Verboom; El Pardo, 10 de marzo de 1742. AGS.SM, 38/95. 